\title{
A Dynamic Skull Model for Simulation of Cerebral Cortex Folding
}

\author{
Hanbo Chen ${ }^{1}$, Lei Guo ${ }^{1}$, Jingxin $\mathrm{Nie}^{1}$, Tuo Zhang ${ }^{1}$, Xintao $\mathrm{Hu}^{1}$, and Tianming Liu ${ }^{2}$ \\ ${ }^{1}$ School of Automation, Northwestern Polytechnical University, Xi' an, China \\ ${ }^{2}$ Department of Computer Science and Bioimaging Research Center, \\ The University of Georgia, Athens, GA, USA
}

\begin{abstract}
The mechanisms of human cerebral cortex folding and their interactions during brain development are largely unknown, partly due to the difficulties in biological experiments and data acquisition for the developing fetus brain. Computational modeling and simulation provide a novel approach to the understanding of cortex folding processes in normal or aberrant neurodevelopment. Based on our recently developed computational model of the cerebral cortex folding using neuronal growth model and mechanical skull constraint, this paper presents a computational dynamic model of the brain skull that regulates the cortical folding simulation. Our simulation results show that the dynamic skull model is more biologically realistic and significantly improves our cortical folding simulation results. This work provides further computational support to the hypothesis that skull is an important regulator of cortical folding.
\end{abstract}

Keywords: Cortex Folding, Simulation, Skull Constraint.

\section{Introduction}

The folding pattern of the cerebral cortex varies greatly between individuals [1], and it is believed to have certain relationship with the brain's cytoarchitecture and functional regionalization [2]. The factors that cause these differences have intrigued neuroscientists for a long period. Due to the difficulties in biological experiment and data acquisition for the developing fetus brain, there are growing efforts in the area of computational modeling and simulation that aim to understand the mechanisms of cortical folding. For example, Raghavan et al. proposed a continuum mechanics-based model of growth to synthesize cortical shapes by using physical laws [3]. Toro et al. proposed a computational morphogenetic model to study the fundamental mechanisms of cortical folding [4]. In our recent work [5], by performing a 3D morphogenetic model, we demonstrated that folding pattern is dependent on mechanical constraints of skull, cell growth rate, and initial geometry of the cortex [5]. We applied surface modeling to simulate the cerebral cortex morphogenesis and use a static skull model to constrain the growth of cerebral cortex.

In this paper, a dynamic skull model is developed to provide more biologically meaningful boundary condition for the cortical folding simulation. Mechanical constraint was the first major factor considered when investigating the determinants of cortical folding [6]. The hypothesis is that since the cortical area is almost three times 
larger than the cranial area, the cortex had to convolve to fit into a relatively small cranial volume. In previous skull models such as the ones in [3] and [5], the constraint force acts on the surface only when it touches the skull. While in this work, the skull constraint also considers the cerebrospinal fluid (CSF) pressure, as illustrated in Fig.1. Instead of using a cranial skull, we applied a dynamic growing volume to constrain the growth of cortex. Since the skull develops with the brain, it is more reasonable to model and simulate a dynamic growth skull constraint. The experimental results in this paper show that the dynamic skull model improves the cortical folding simulation significantly.

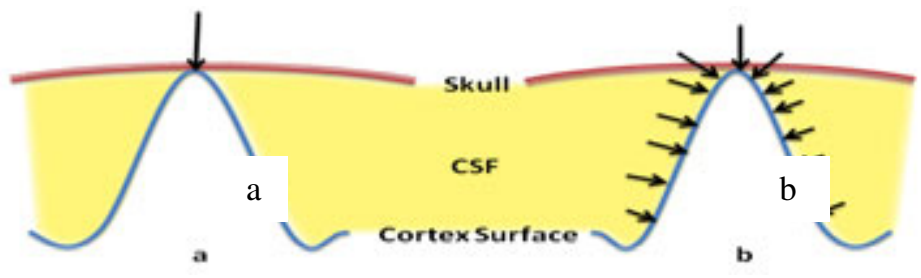

Fig. 1. (a) In previous skull models, constraint only acts on cortex surface when it touches the skull. (b) As the cortex is surrounded by CSF, the CSF pressure is also considered.

\section{Method}

The flowchart of our model and simulation is outlined in Fig.2. We reconstruct cortical surface from diffusion tensor imaging (DTI) data [7] and decompose it into multiresolution representations via the spherical wavelet method [8]. The smooth cortical surface of the lowest resolution is used as the synthesized fetus cortex for the folding

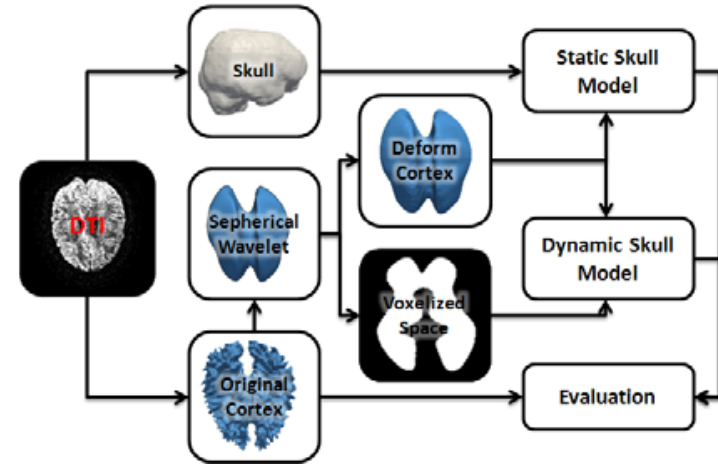

Fig. 2. The flowchart of the model and simulation simulation. The folding simulation is driven by the neuronal growth model in [5] and regulated by the proposed dynamic skull model. The cortical shapes generated by the simulation models are evaluated by quantitative descriptors of curvature, parametric cortical folding measurement [9], and surface distance. It is noted that DTI data, instead of T1 structural data, is used here for surface reconstruction because this will facilitate our future investigation of how axonal fibers regulate the cortical folding.

\subsection{Materials and Pre-processing}

Since the acquisition of fetus brain imaging data is quite challenging, we synthesize the fetus brain surface as follows. The original cortical surface was reconstructed from DTI data of adult brains using the DTI-based tissue segmentation [7] and the cortical surface reconstruction method in [10]. Then, we decompose the cortical surface into multi-resolution representations via the spherical wavelet algorithm [8], 
in which the highly convoluted and complex cortical surface is then decomposed into a cascade of lower-resolution surfaces, as shown in Fig.3(a-e). Fig.3(f) shows a fetus cortical surface reconstructed from fetus MRI data [5]. We can see that the lowresolution surface (Fig.3(e)) appears to be similar to the fetus cortex in Fig.3(f). This visualization supports the correctness of our method for synthesizing fetus cortical surface. Moreover, in this way, we are able to compare the simulation results with original surface to evaluate the model.

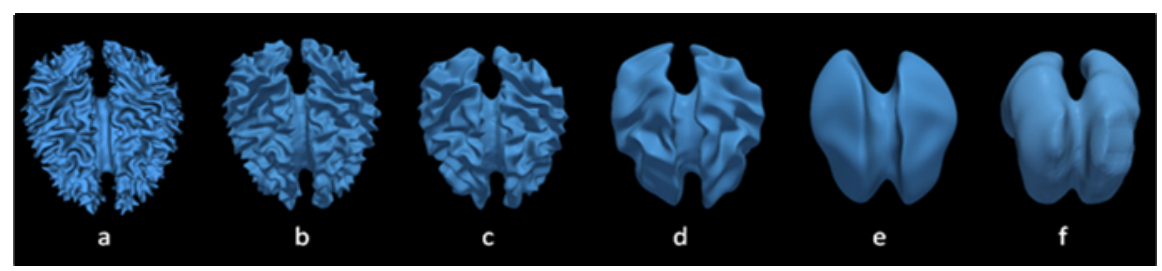

Fig. 3. (a) Original surface generated from DTI data. (b)-(e) Multi-resolution decomposition of cortical surface (the fifth, forth, third and second resolutions). (f) Cortical surface of fetus brain reconstructed from real MRI data [5].

Notably, the surface decomposition method [8] might produce unbalanced surface at low resolution, in which triangles in certain region might be much smaller than other regions as shown in Fig. 4 or intersect with other triangles. Thus, to obtain a balanced initial surface, we voxelized the low-resolution surface into a volumetric image, and reconstructed a new surface using marching cube algorithm.

\subsection{Static Skull}

The development of the cerebral cortex is constrained by the cranial volume. We developed a volumetric constraint model [5] to simulate the effect of static skull during the folding of the cortex, as illustrated in Fig.5. When any vertex of the surface is being deformed to a new position $\mathbf{x}^{\prime}$, the following condition should be satisfied: the new position of the vertex cannot intersect with

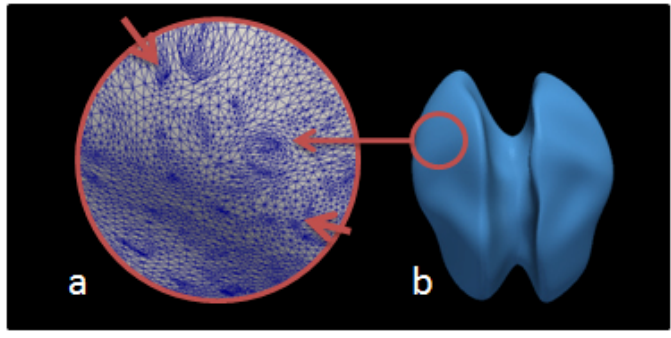

Fig. 4. The flowchart of deformable surface initialization. (a) Second resolution of the decomposed surface. (b) Voxelized surface. (c) Deformable surface. (d) Zoomed-in view of the surface patch in the circle in (a).

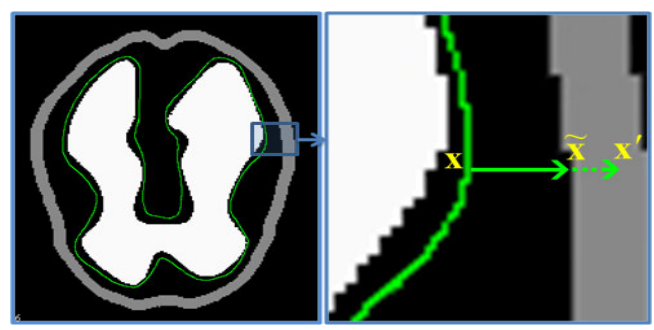

Fig. 5. The condition that constrains the development of cortex. When cortex point $\mathbf{x}$ is trying to deform to the new position $\mathbf{x}^{\prime}$, the following condition should be satisfied: $\mathbf{x}^{\prime}$ should not intersect with other brain tissues. 
cranial volume. If the condition is not satisfied, another new position $\widetilde{\mathbf{x}}$ for this vertex that satisfies the condition should be identified. After finding the new position satisfying the conditions, the vertex can be deformed to the new position.

\subsection{Dynamic Skull}

Since skull is developing during the cortical folding, its growth should be modeled dynamically. Thus, the development of skull that is caused by the outgrowth of cortex is considered in our paper, and the dynamic growth of skull is defined as follows. Similar to the volumetric constraint model in Fig.5, a constraint function $L(n)$ is defined on each voxel in the deformable space, where $n$ is the iteration number. When $L(n) \leq 0$, the voxel is defined as a deformable voxel, which cortex surface could deform into at iteration $n$, otherwise it is defined as constraint voxel. When $n=0, L(n)$ is the initial skull constraint in which zero value space is defined as inner space of initial cortical surface in our experiments. When $n>0, L(n)$ is interacting with the cortex folding process by:

$$
L(n)=L(0)-\lambda_{L} \sum_{n} I(n)
$$

where $\lambda_{L}$ controls the growth speed of the skull and $I(n)$ is defined on each voxel. At iteration $n$, if any vertex or triangle on the cortex surface is trying to deform to a voxel, the value of $I(n)$ in this voxel is set as one, otherwise it is zero, meaning that the attempt of the cortex deformation into skull voxel will cause the growth of intracranial volume.

In this model, the constraint not only acts on the top of gyrus, but also on the lateral surface. The cortical surface is encircled by constraint voxel, thus when it grows to the outer space, its movement will be limited. This effect is the same as fluid pressure. As shown in Fig.1, because brains are surrounded by CSF, the constraint pressure should take the CSF pressure into consideration.

\subsection{Development of the Cerebral Cortex}

We used the models in [5] to simulate the development of the cerebral cortex. The developed model is composed of the following four key components.

a) The deformable model. The elasto-plasticity property is adopted on each edge of the triangle in the surface. Since the surface could be considered as a zero thickness sheet, the bending energy is introduced to model the rigidity of cortex.

b) The growth model. Growth of cerebral cortex is defined on each triangle of the surface. The classic logistic-growth function is adopted to describe the growth of cortical tissues as:

$$
\frac{d A_{c 0}}{d t}=A_{c 0} m\left(1-\frac{A_{c 0}}{k}\right)
$$

where $\mathrm{m}$ is known as the Malthusian parameter, $\mathrm{k}$ is the carrying capacity of the system and $A_{c 0}$ is the rest area of triangle. 
c) Constraint model. The development of cortex is limited by the boundary condition of cranial volume.

d) Model solver. The proposed model can be formulated as a time varying partial differential equation. The explicit Newmark scheme is adopted to solve the model.

\section{Experimental Results}

In this section, we simulate the proposed computational model of cortical folding with static and dynamic skull constraints, and compare the results. Furthermore, we compare the simulations with different cortical growth rates.

\subsection{Comparison of Two Skull Models}

The values of parameters in the growth model are the same in the static skull model and dynamic skull model: $\lambda_{L}=0.1, m=0.009, k=3$. The results of folding development are illustrated in Fig.6. Fig.6(a-b) show the snapshots of cortex development at the iteration number 50,100, 150, and 200 in static skull model and dynamic skull model, respectively. By visual evaluation, it is evident that more realistic folding is generated using the dynamic skull model. To quantify this difference, we use the average absolute Gaussian curvature and parametric folding pattern [9] as shape descriptor to evaluate the produced convolutions. We also use the distance between original surface and deformed surface to evaluate the simulation models. The differences between the average absolute Gaussian curvatures, folding pattern descriptors of the cortex surface in different simulation iterations, and the surface distances of five cases we simulated are illustrated in Fig.7(a-c) respectively. It is evident that the folding patterns produced by the dynamic skull models are much closer to those of normal brain surfaces obtained from real MRI images. It is also intriguing that when iteration number reaches certain steps, the average absolute Gaussian curvature of cortical surface will reach a steady condition. This steady condition is mainly because of the fact that after the cortex convoluted completely, the cortex will only develop vertically and thus curvatures do not change much. Fig.7(a) shows that the dynamic skull model needs only half of the iteration time of the static skull model to reach the same average curvature.

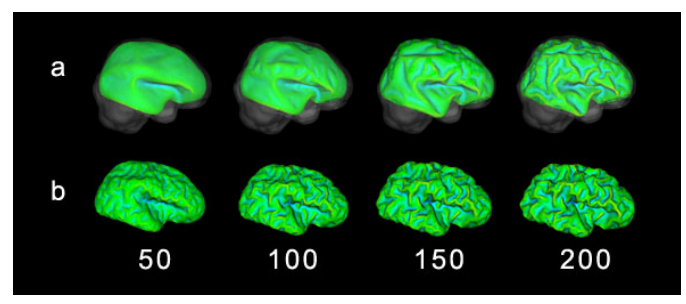

Fig. 6. Demonstrations of cortical development results: (a) Static skull model. (b) Dynamic skull model. (a)-(b) show the snapshots of cortex at the iteration number $50,100,150$, and 200 respectively. A video of this progress is affiliated in supplementary materials.

\subsection{Comparison of Growth Rates}

In this section, we investigate the effect of skull growth rate on the cortical folding process using the dynamic skull model. Three groups of simulation were performed 


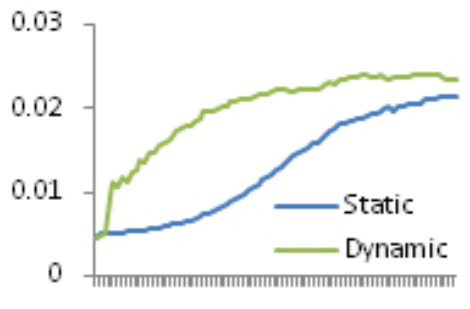

$0 \quad 50100150200250300350$

(a)

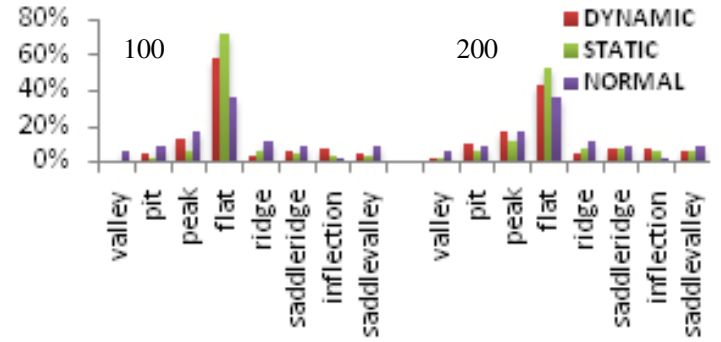

(b)

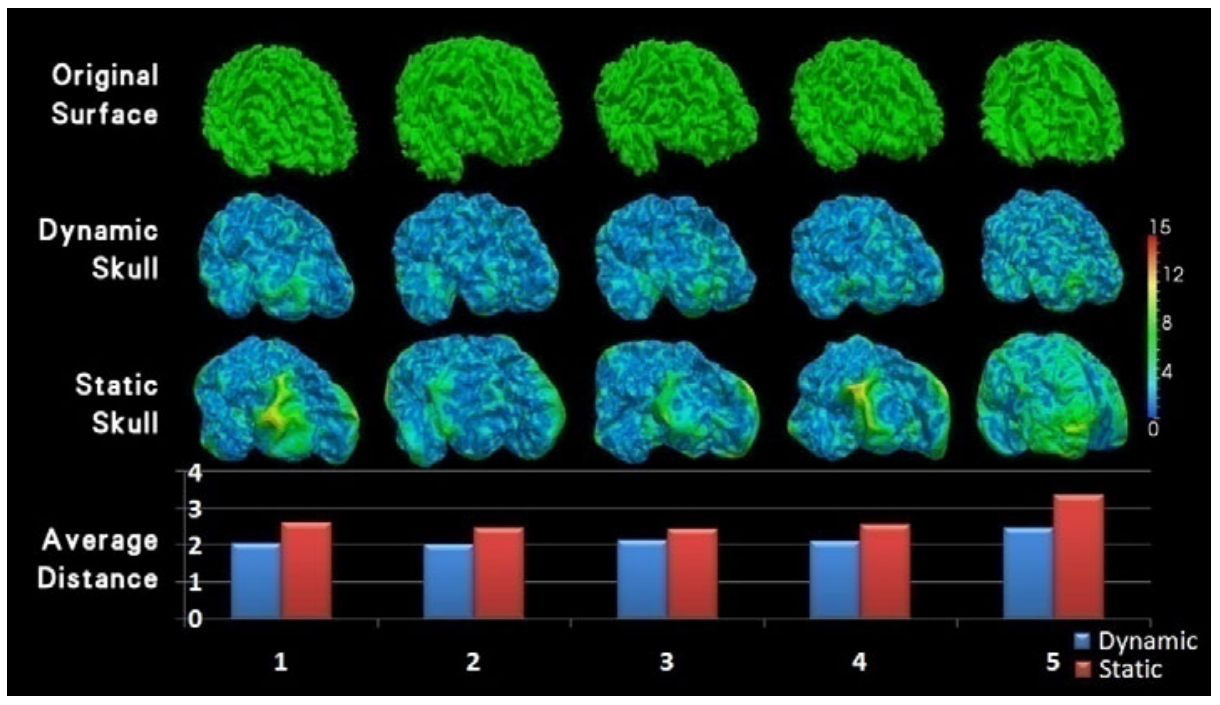

(c)

Fig. 7. (a) The difference of the average absolute Gaussian curvature of the cortex surface during simulated growth. (b) Folding pattern distributions at 100 and 200 iterations. More details of the folding descriptors are referred to [9]. (c) The distance between deformed surface and original surface for both dynamic and static skull models. The color bar is on the right.

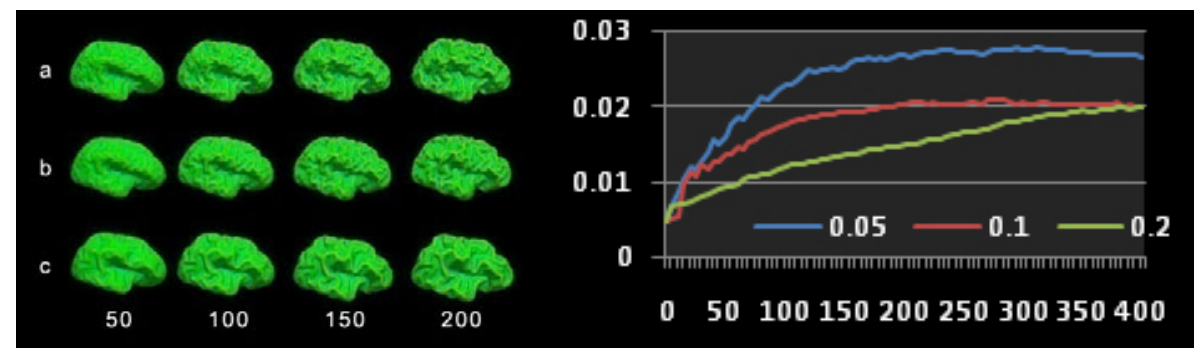

(a)

(b)

Fig. 8. (a) Cortical developing in different skull growth rates. a: $\lambda_{L}=0.05$; b: $\lambda_{L}=0.1$; c: $\lambda_{L}=0.2$. (b) The difference of the average absolute Gaussian curvature of the developing surface with different $\lambda_{L}$. 
with different $\lambda_{L}$ arrange from 0.05 to 0.2 , which present slow to fast growth rate respectively. As shown in Fig.8, slower skull growth rates can generate more convolutions. A slower growth rate means smaller space for cortex to grow at the same time and thus more constraint force. By changing growth rate of dynamic skull, we can simulate condition with different constraint force - the slower the growth rate is, the higher the constraint force will be. This result further indicated that skull constraint is an important regulator of the cortical folding process.

\section{Discussion and Conclusion}

Our simulations demonstrate that mechanical constraints imposed by the skull are important regulators of cortical folding. However, it should be noted that our simulations indicate that skull constraint is not necessarily the dominant or initializing mechanism. Previous experimental observation has also shown that it is difficult to conclude that skull restraint initiates the development of cortical folding [11]. Our current model of neuronal growth also assumes isometric deformation. However, it should be noted that this isometric model is insufficient. In [12], it was demonstrated that there are systematic variations in absolute thickness and cell number across the cortical landscape, e.g., the gyral columns contain significantly more neurons than sulcal columns. Currently, the growth parameter for our simulations are either homogenous or set differently in manually selected regions. In future work we plan to infer or estimate growth parameters from MRI data of the developing brain by measuring cortical thickness or gray matter density. The cortical measurements will then be mapped to the simulation space via cortical surface registration algorithms allowing us to investigate how multiple, locally intrinsic and real differentiations influence the cortical folding process.

In this paper, we present a dynamic skull growth model for improved simulation of cerebral cortex folding. Our results show significant improvement by the dynamic model. In our current model, only neuronal growth and dynamic skull constraints are modeled and simulated. In future, we intend to examine, model and simulate more premises regarding cortex folding mechanisms. For instance, as previously stated in the tension-based theory of morphogenesis [13], cortical patterns are considered to be the result of minimization of global tension energy along axons, dendrites, and neuroglia. Our future work will extend the computational system to a more general framework that could easily plug-in new mechanical force models, implemented according to different biological premises such as the tension-based morphogenesis theory [13]. By using above approach, different computational models of cortical folding mechanisms could be independently developed, and could be easily integrated to study their interactions. After reaching such a stage, it would be possible to simulate more realistic cortical folding patterns that are similar to those of a real, human cerebral cortex.

\section{References}

1. Talairach, J., Tournoux, P.: Co-planar Stereotaxic Atlas of the Human Brain. Thieme, New York (1988)

2. Fischl, B., et al.: Cortical Folding Patterns and Predicting Cytoarchitecture. Cereb Cortex 18(8), 1973-1980 (2008) 
3. Raghavan, R., et al.: A continuum mechanics-based model for cortical Growth. J. Theor. Biol. 187, 285-296 (1997)

4. Toro, R., Burnod, Y.: A Morphogenetic Model for the Development of Cortical Convolutions. Cerebral Cortex 15, 1900-1913 (2005)

5. Nie, J., et al.: A Computational Model of Cerebral Cortex Folding. In: Yang, G.-Z., Hawkes, D., Rueckert, D., Noble, A., Taylor, C. (eds.) MICCAI 2009. LNCS, vol. 5762, pp. 458-465. Springer, Heidelberg (2009)

6. Le Gros Clark, W.: Deformation patterns on the cerebral cortex. In: Essays on Growth and Form, pp. 1-23. Oxford University Press, Oxford (1945)

7. Liu, T., et al.: Brain Tissue Segmentation Based on DTI Data. NeuroImage 38(1), 114-123 (2007)

8. Yeo, B.T.T., et al.: Shape Analysis with Overcomplete Spherical Wavelets. In: Metaxas, D., Axel, L., Fichtinger, G., Székely, G., et al. (eds.) MICCAI 2008, Part I. LNCS, vol. 5241, pp. 468-476. Springer, Heidelberg (2008)

9. Zhang, T., et al.: Parametric representation of cortical surface folding based on polynomials. In: Yang, G.-Z., Hawkes, D., Rueckert, D., Noble, A., Taylor, C., et al. (eds.) MICCAI 2009. LNCS, vol. 5762, pp. 184-191. Springer, Heidelberg (2009)

10. Liu, T., et al.: Deformable Registration of Cortical Structures via Hybrid Volumetric and Surface Warping. NeuroImage 22(4), 1790-1801 (2004)

11. Barron, D.: An experimental analysis of some factors involved in the development of the fissure pattern of the cerebral cortex. J. Exp. Zool. 113, 553-581 (1950)

12. Hilgetag, C.C., et al.: Developmental mechanics of the primate cerebral cortex. Anat. Embryol. 210, 411-417 (2005)

13. Van Essen, D.C.: A tension-based theory of morphogenesis and compact wiring in the central nervous system. Nature (1997) 